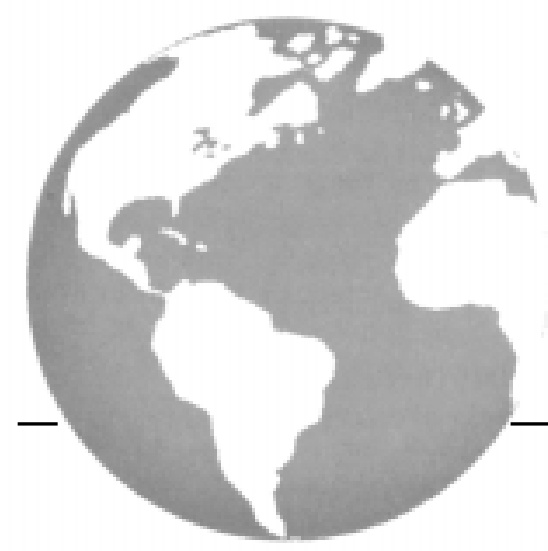

\title{
Solitary bone metastasis beneath the shoulder shield: coincidence or cause?
}

\author{
G. Fan BSc (C), E. Sinclair MRT (T), \\ M. Christakis $M D$, L. Erhlich $M D$, \\ J. Zubovits $M D$, and E. Chow MBBS
}

\begin{abstract}
Post-mastectomy radiotherapy has been demonstrated to improve locoregional control in breast cancer patients. We report a case involving a 44-year-old breast cancer patient who presented with a solitary bone metastasis in the area beneath the shoulder shield, likely from a coincidental recurrence.
\end{abstract}

\section{KEY WORDS}

Solitary bone metastasis, recurrence, post-mastectomy radiotherapy

\section{INTRODUCTION}

Post-mastectomy radiotherapy has been demonstrated in randomized controlled trials to improve locoregional control in breast cancer patients ${ }^{1,2}$. However, even after radiation treatment, 10\%-30\% of patients relapse locoregionally, $60 \%-70 \%$ relapse distally, and $10 \%-30 \%$ experience both types of relapse $^{3}$.

At our centre, when radiating the breast, it is standard practice to employ a shoulder shield to protect normal tissue. Here, we report a post-radiotherapy case of a solitary bone metastasis in the area beneath the shoulder shield in a breast cancer patient.

\section{CASE HISTORY}

A 44-year-old woman presented with a self-detected lump in her left breast. She underwent a left modified radical mastectomy, and pathology reviewed a $4.5-\mathrm{cm}$ grade II infiltrating ductal carcinoma with 9 of 16 lymph nodes involved. The tumour was progesterone-positive but estrogen- and HER 2/neu-negative. The metastatic work-up, including a bone scan, was negative. The patient was treated with dose-dense doxorubicin-cyclophosphamide/paclitaxel and with radiation to locoregional lymphatic-bearing areas in an adjuvant fashion. During the radiation treatment, a shield was employed to protect the patient's left shoulder (Figure 1).

A rheumatologist followed the patient because of ongoing benign sciatic pain. Because of increased pelvic pain, a bone scan and computed tomography (Ст) scan were ordered. The imaging showed erosive changes in the right sacroiliac joint, associated with sclerosis of the adjacent bone-consistent with sacroiliitis. In addition, the bone scan revealed an increased uptake in the left shoulder (Figure 2). A CT scan and a radiograph of that area confirmed a $2.5-\mathrm{cm}$ region of sclerosis within the left humeral head, representing bone metastasis (Figure 3).

Under CT guidance, a biopsy of the left humeral head was performed, and pathology confirmed metastatic carcinoma consistent with a breast primary

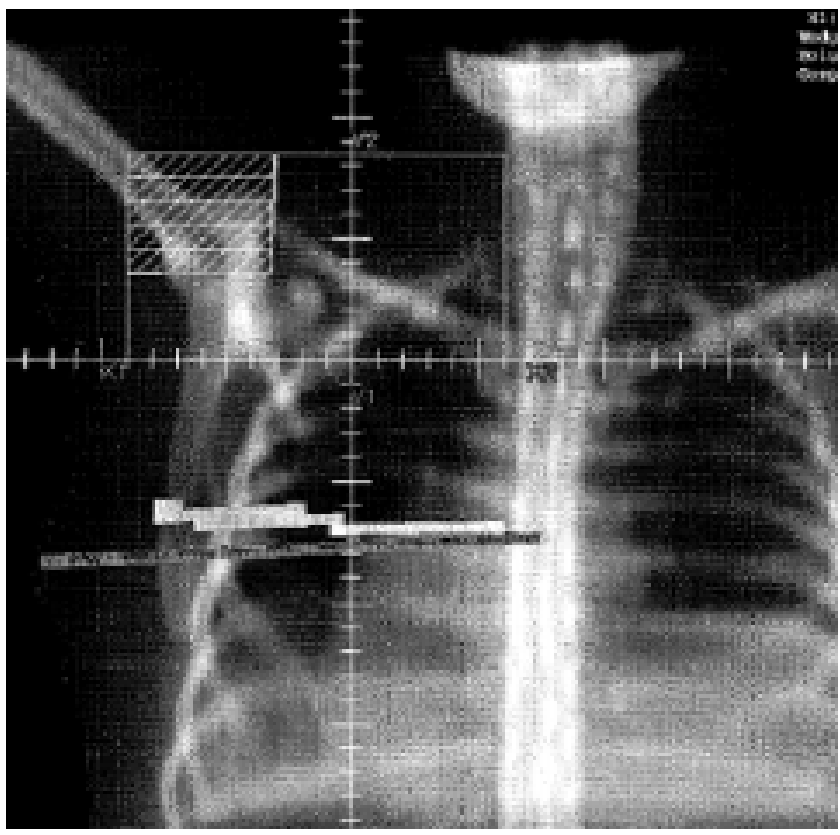

FIGURE 1 A shield was employed over the humeral head of the left shoulder during the radiation treatment. 


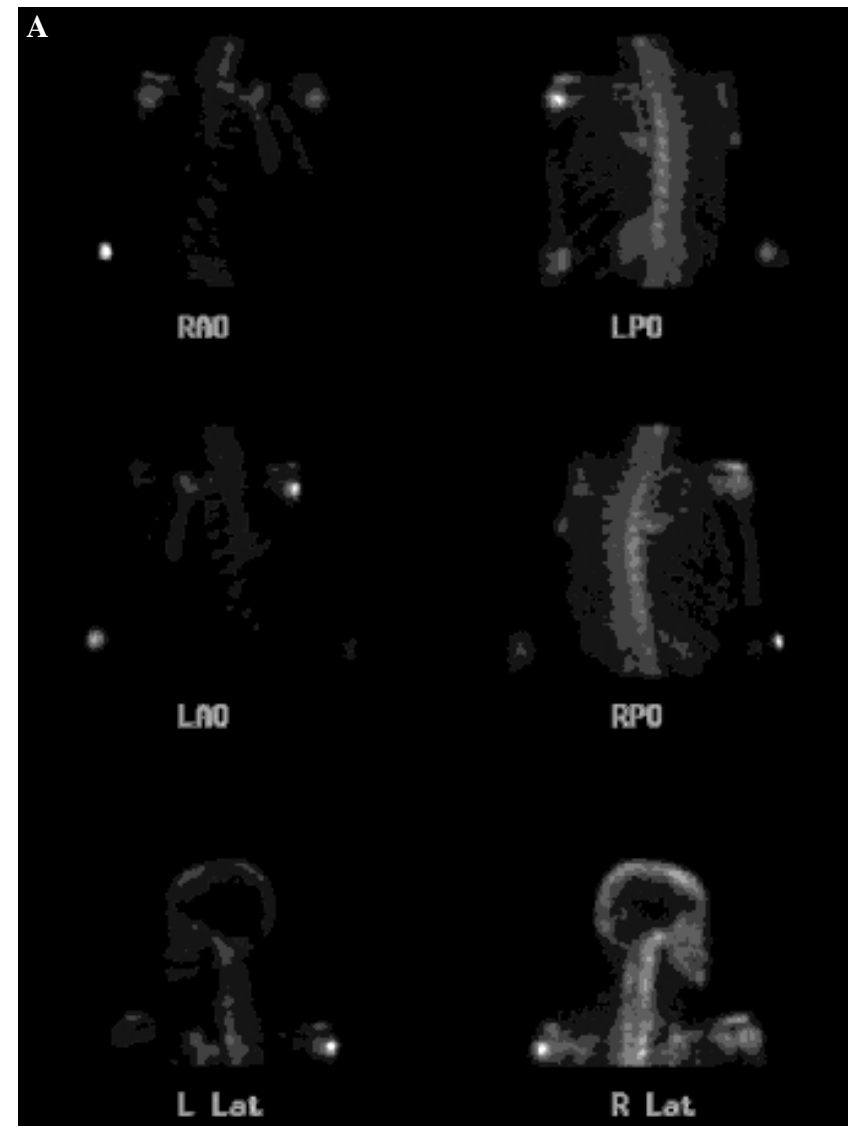

B

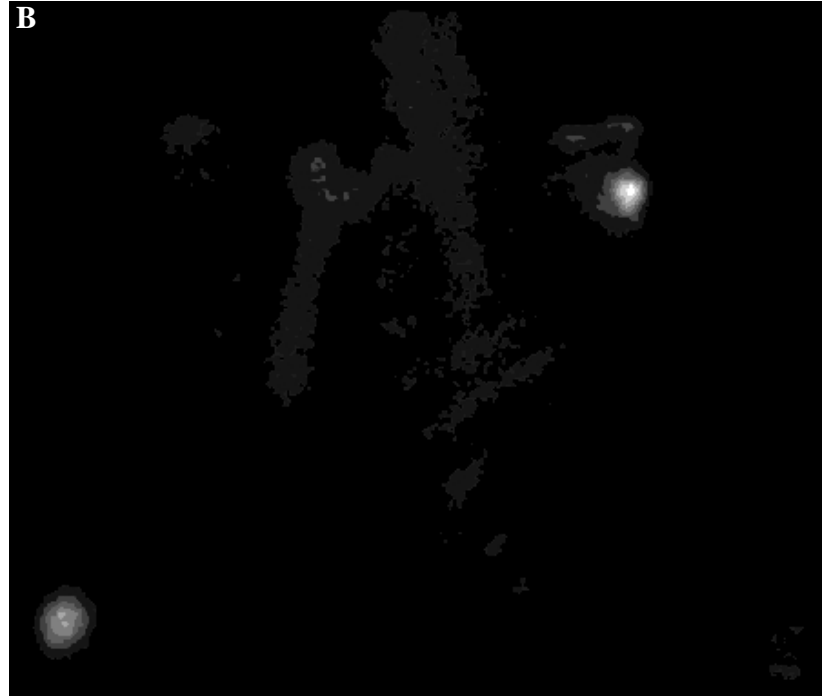

FIGURE 2 A technetium methylene diphosphonate bone scan showing increased tracer uptake in the posterior aspect of the left humeral head. (A) Left/right anterior/posterior oblique and lateral views. (B) Enlargement of left anterior oblique view.

(Figure 4). The patient had a repeat metastatic workup, but showed no confirmed evidence of visceral involvement at the time of writing. She was treated with bisphosphonates and palliative radiotherapy for the bone metastasis in the left shoulder.
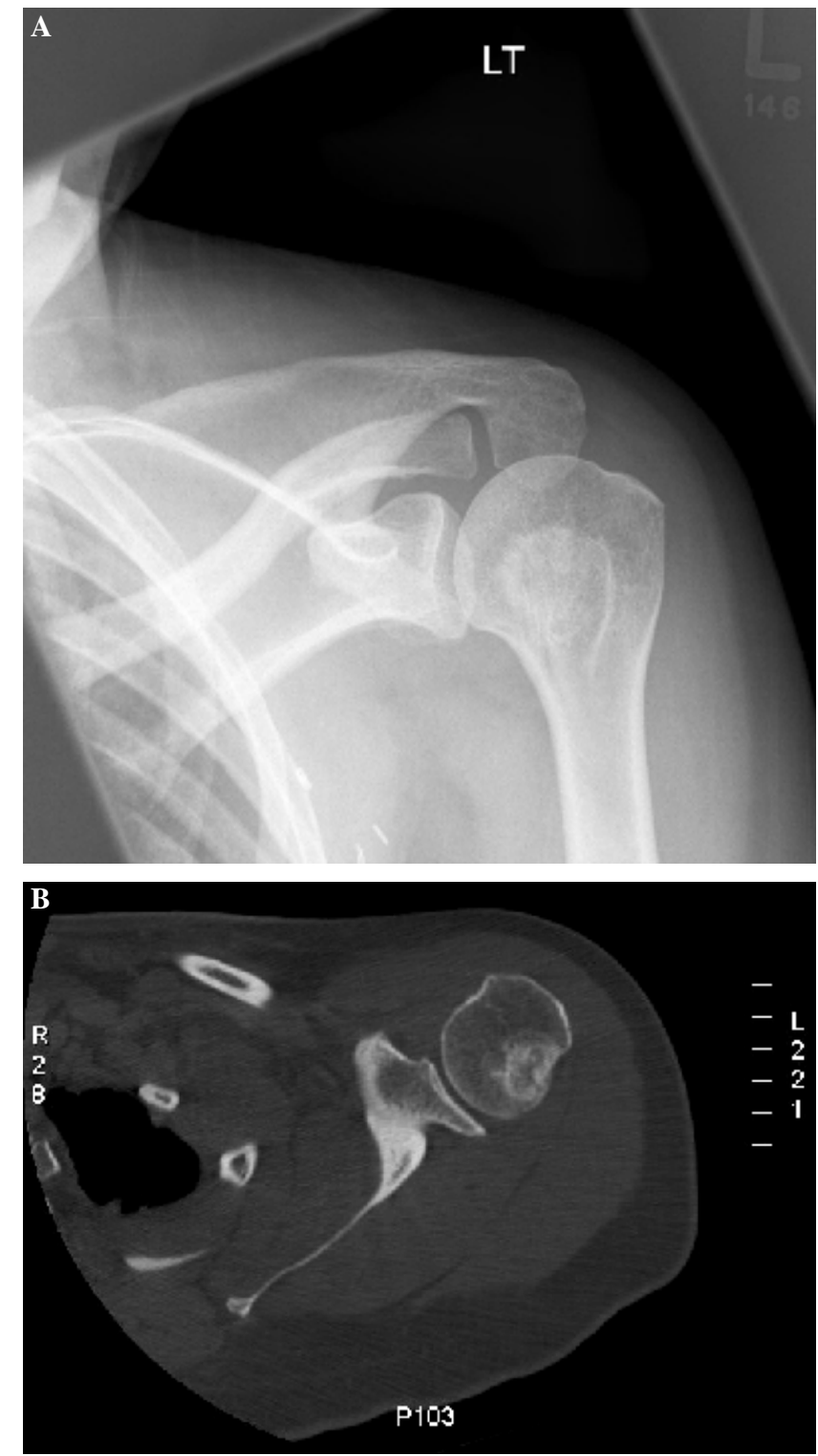

FIGURE 3 (A) Anterior/posterior radiograph of the left shoulder demonstrates a sclerotic lesion in the humeral neck corresponding to the abnormality on the bone scan. (B) Axial computed tomography image of the left shoulder confirms a predominantly sclerotic $1.9-\mathrm{cm} \times 1.8 \mathrm{~cm} \times 2.0-\mathrm{cm}$ eccentric lesion in the posterolateral humeral neck, in keeping with a metastatic focus.

\section{DISCUSSION AND CONCLUSIONS}

This unfortunate woman was at risk of distant metastasis. At present, she has developed a solitary bone metastasis in the left shoulder corresponding to the shoulder shield in the radiation treatment.

Recurrence features in more than two thirds of breast cancer patients treated with post-mastectomy radiotherapy (3). Lee found that the greater the extent of axillary metastases, the higher the relapse rate. When axillary nodes are unremarkable, $18 \%$ of pa- 

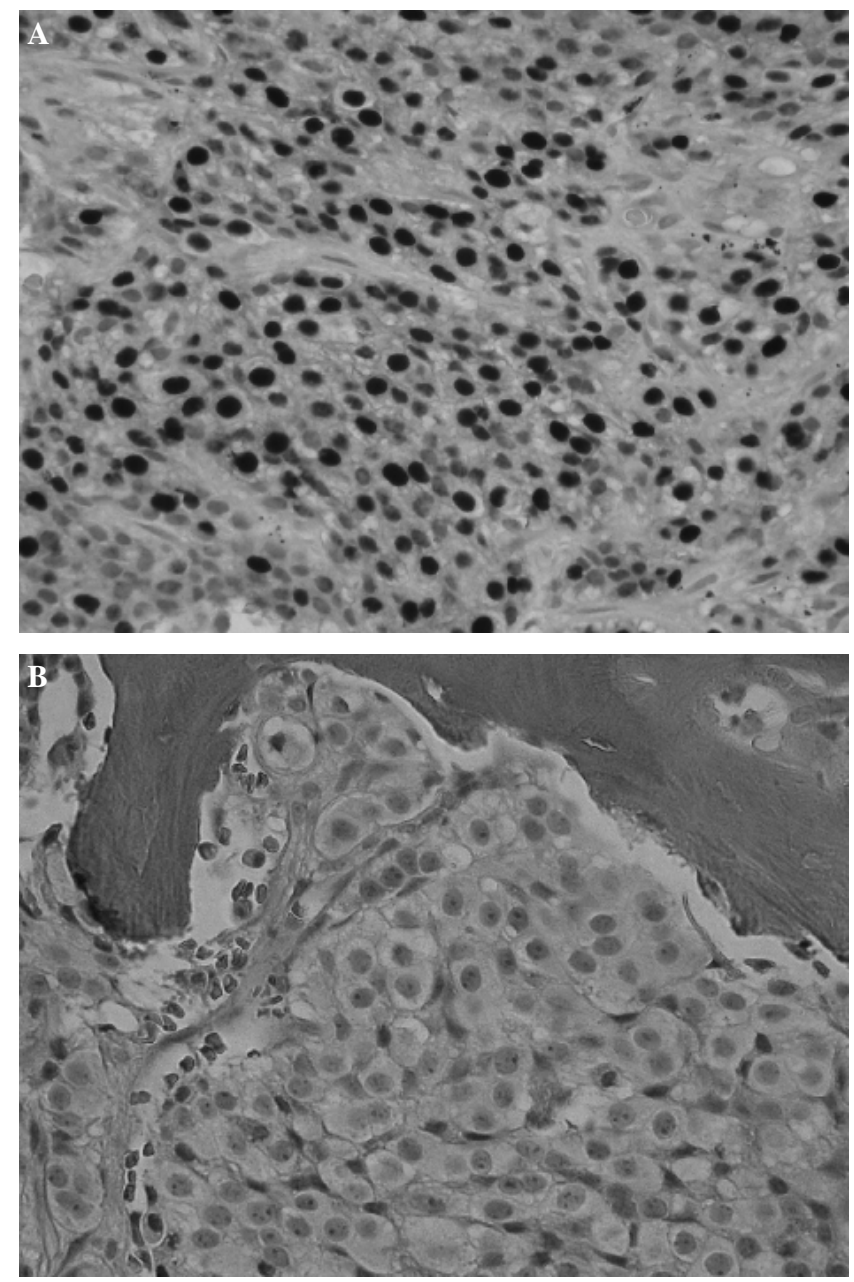

FIGURE 4 (A) Bone biopsy of the humerus shows large malignant epithelioid tumour cells growing in nests and sheets within spicules of bone. The appearance is consistent with a breast primary. (B) The tumour cells show strong staining for the progesterone receptor (consistent with a breast primary) and the same reactivity as the primary tumour.

tients experience recurrence. Patients with 1-3 nodes and more than 4 nodes positive relapse at rates of $24 \%$ and $69 \%$ respectively (3). Bone is the main site of recurrence and accounts for $40 \%-60 \%$ of distant metastases (3).

Metastasis to the bone is common in breast cancer, arising in $20 \%-60 \%$ of patients and in up to $70 \%-$ $85 \%$ at autopsy (4-7). More than $69 \%$ of these patients have a solitary recurrence confined to a single anatomic site, and $12 \%$ have multiple metastatic sites (8). However, only $3.5 \%$ of breast cancer patients develop long-bone metastases, and of those, $88 \%$ experience recurrence in the femur (9). When the humerus is involved, almost $90 \%$ of lesions arise in the proximal $(42 \%)$ and diaphyseal $(47 \%)$ regions (10). Metastases to the distal humerus are more infrequent, accounting for only $11 \%$ of cases (10).

The location of the metastasis in our patient was initially a curious surprise. Did the treatment protocol play a part in the area of metastasis? Or was it a coincidence that the bone lesion developed in the exact area shielded with the intent of protecting normal tissue? Most likely, the location of recurrence is a mere coincidence.

\section{REFERENCES}

1. Early Breast Cancer Trialists' Collaborative Group. Effects of radiotherapy and surgery in early breast cancer. An overview of the randomized trials. $N$ Engl J Med 1995;333:1444-55. [Erratum in N Engl J Med 1996;334:1003]

2. Early Breast Cancer Trialists' Collaborative Group. Favourable and unfavourable effects on long-term survival of radiotherapy for early breast cancer: an overview of the randomised trials. Lancet 2000;355:1757-70.

3. Lee YT. Breast carcinoma: pattern of recurrence and metastasis after mastectomy. Am J Clin Oncol 1984;7:443-9.

4. Beals RK, Lawton GD, Snell WE. Prophylactic internal fixation of the femur in metastatic breast cancer. Cancer 1971; 28:1350-4.

5. Abrams HL, Spiro R, Goldstein N. Metastases in carcinoma; analysis of 1000 autopsied cases. Cancer 1950;3:74-85.

6. Habermann ET, Sachs R, Stern RE, Hirsh DM, Anderson WJ Jr. The pathology and treatment of metastatic disease of the femur. Clin Orthop Relat Res 1982;169:70-82.

7. Tubiana-Hulin M. Incidence, prevalence and distribution of bone metastases. Bone 1991;12(suppl 1):S9-10.

8. Kamby C, Ejlertsen B, Andersen J, et al. The pattern of metastases in human breast cancer. Influence of systemic adjuvant therapy and impact on survival. Acta Oncol 1988;27: 715-19.

9. Knutson CO, Spratt JS Jr. The natural history and management of mammary cancer metastatic to the femur. Cancer 1970; 26:1199-203.

10. Thai DM, Kitagawa Y, Choong PF. Outcome of surgical management of bony metastases to the humerus and shoulder girdle: a retrospective analysis of 93 patients. Int Semin Surg Oncol 2006;3:5.

Correspondence to: Edward Chow, Department of Radiation Oncology, Toronto Sunnybrook Regional Cancer Centre, 2075 Bayview Avenue, Toronto, Ontario M4N 3M5.

E-mail: edward.chow@sunnybrook.ca 